\title{
Review Article \\ Innate Immune Evasion Strategies by Human Immunodeficiency Virus Type 1
}

\author{
Debjani Guha and Velpandi Ayyavoo \\ Department of Infectious Diseases and Microbiology, Graduate School of Public Health, University of Pittsburgh, 130 Desoto Street, \\ Pittsburgh, PA 15261, USA
}

Correspondence should be addressed to Velpandi Ayyavoo; velpandi@pitt.edu

Received 30 May 2013; Accepted 9 July 2013

Academic Editors: B. Joos, H. C. Mwandumba, and C. Petrovas

Copyright (C) 2013 D. Guha and V. Ayyavoo. This is an open access article distributed under the Creative Commons Attribution License, which permits unrestricted use, distribution, and reproduction in any medium, provided the original work is properly cited.

\begin{abstract}
Host immune components play both beneficial and pathogenic roles in human immunodeficiency virus type 1 (HIV-1) infection. During the initial stage of viral infection, a complex network of innate immune factors are activated. For instance, the immune cells express a number of inflammatory proteins including cytokines, chemokines, and antiviral restriction factors. These factors, specifically, interferons (IFNs) play a crucial role in antiviral defense system by modulating the downstream signaling events, by inducing maturation of dendritic cells (DCs), and by activation of macrophages, natural killer (NK) cells, and B and T cells. However, HIV-1 has evolved to utilize a number of strategies to overcome the antiviral effects of the host innate immune system. This review discusses the pathways and strategies utilized by HIV-1 to establish latent and persistent infection by defeating host's innate defense system.
\end{abstract}

\section{Introduction}

During the early phase of infection, hosts mount innate immune response that comprises defense mechanisms to protect the hosts from invading pathogens in an antigen independent manner. This immune response is the first and a rapid response launched against a variety of microorganisms. The innate immune system can distinguish between self and foreign proteins and responds accordingly. This nonspecific immune response is activated primarily by the structural motifs of invading pathogens. The major cell types that play key roles in innate immune response against invading pathogens include macrophages, dendritic cells, neutrophils, natural killer cells, mast cells, eosinophils, and basophils. Most of the innate effector cells produce inflammatory factors that function as chemical messengers. Among these molecules, IFNs are the most effective in elucidating antiviral immune responses [1]. Additionally, cytokines and chemokines also play important roles as chemoattractants controlling leukocytes trafficking. Innate immune response operates through the steps of recognition of the pathogen, signal transduction, and subsequent gene expression to produce the innate immune effector molecules.

The first step is to recognize a pathogen as a foreign object and differentiate it from self-components. When pathogens breach physical barriers such as the skin or oral mucosa, they are recognized by pattern recognition receptors (PRRs) expressed either in the cytoplasm or on cell membranes. PRRs sense and interact with the structurally conserved motifs of proteins and nucleic acids unique to invading pathogens known as pathogen-associated molecular patterns (PAMPs) [2]. The most widely studied PRRs, the toll-like receptors (TLRs), present either on the cell surface or in the endoplasmic compartments, are involved in recognizing microbial PAMPs. For example, TLR2 and TLR4 respond to specific viral glycoproteins; TLR9, TLR3/7, and TLR8 are involved in sensing viral nucleic acids as well as the unmethylated $\mathrm{CpG}$ sequence in viral DNA molecules [3]. In addition to TLRs, viral PAMPs are also detected by other PRRs including RIG-like receptors (RLRs), RIG-I, MDA5, C-type lectin receptor (CLR), and DC-SIGN. RIG-I and MDA5 recognize $5^{\prime}$ phosphorylated short and long dsRNA, 
respectively, whereas, DC-SIGN binds to viral envelop glycoproteins. Cytosolic receptors such as AIM2 and DAI are also identified as respondents of dsDNA [4]. The interaction of viral ligands with host receptors activates the downstream signaling events that in turn switch on specific transcription factors regulating the expression of genes responsible for innate and adaptive immunity interchange. For example, when TLRs bind to viral PAMPs, the intracellular part of TLR binds to MyD88 and activates mitogen activated protein kinase (MAPK) that leads to the activation of NF- $\kappa \mathrm{B}$. Activation of NF- $\kappa$ B promotes regulation of inflammatory cytokine genes and activates interferon regulatory factor (IRF) [5, 6]. IRF induces type I IFNs that function as antiviral and inflammatory agents [7]. Furthermore, IFN is also involved in maturation of DC and regulates the function of macrophages, NK cells, and T and B cells [8]. However, the efficacy of host response depends on a rapid and specific recognition response to invading pathogens. Upon infection, immune cells are activated to modulate their molecular networks to eliminate the pathogen.

\section{Strategies of HIV-1 to Evade Innate Immune Response}

To overcome these immune effector functions, viruses including retroviruses are evolved to counteract and subvert through various mechanisms $[9,10]$. Retroviruses are a diverse family of enveloped RNA viruses that have the ability to evade immune defense system and establish long-term persistence in the infected hosts. HIV-1, one of the retroviruses, utilizes varying strategies compared to other viruses as the PAMPs exposed by the virus are not easily recognized by the PRRs, and hence the virus can escape from proinflammatory and antiviral responses [11]. One possible mechanism by which HIV-1 manages to avoid immune encounter of the host is that HIV-1 can modify its PAMPs by altering or hiding its nucleic acids in the viral capsid in order to mimic the cellular proteins [12]. The ability of genetic variability of HIV-1 is one of the major immune evasion strategies for the virus. The HIV-1 RNA genome can be mutated randomly which helps the virus to evade immune recognition by the host. Error prone viral reverse transcriptase lacking the proofreading activity is responsible for the high mutation rate in HIV-1 [13]. By avoiding the immune recognition, HIV-1 crosses various checkpoints in innate as well as adaptive immune defense machineries of the host. This review emphasizes on some of the mechanisms utilized by HIV-1 to escape host's innate antiviral responses.

\section{Evasion of HIV-1 through Mucosal Barriers during Early Infection}

HIV-1 enters primarily through the mucosal surfaces of genital or rectal tissues during sexual transmission. Mucosa presents the first line of physical defense against invading pathogens. HIV-1 evades this initial barrier by successfully crossing the mucosa to reach the susceptible host cells and establish the infection. One of the proposed mechanisms is that the virus crosses the mucosal barrier by transcytosis or by capturing dendrites of intraepithelial DCs [14]. Additionally, HIV-1 also utilizes the intercellular spaces to move through the epithelium and achieve contact with the underlying mucosal Langerhans cells and $\mathrm{CD}_{4}^{+} \mathrm{T}$ cells [14]. Exposure of HIV-1 or the viral protein gp120 disrupts tight junction proteins and the monolayer integrity of mucosal epithelium by the upregulating inflammatory cytokines that leads to the increased permeability of the virus particles [15]. These studies suggest that HIV-1 may induce defects at the mucosal epithelial barrier, which could activate mucosal $\mathrm{T}$ cells and increase the production of inflammatory cytokines [16-18]. A recent study demonstrated the association of $\gamma \delta \mathrm{T}$ cells as a major component of mucosal immune system with the early HIV-1 induced events [19]. Breakdown of mucosal barrier is considered as the most crucial event causing HIV-1-associated immune activation.

Following mucosal breaching, HIV-1 establishes acute infection in immune cells present within the mucosa. It has been suggested that the productive HIV-1 infection starts from a single infectious virus particle [20,21], and the resting $\mathrm{CD}^{+} \mathrm{T}$ cells are the first targets [21-23]. These infected cells disseminate with the help of proinflammatory cytokines such as IL-1, IL-8, IL-6, and GM-CSF to the lymphoid tissues throughout the body including the gut-associated lymphoid tissue (GALT) containing high numbers of $\mathrm{CD}^{+}$ $\mathrm{T}$ cells where the virus replicates at a very high rate. A group of freshly infected $\mathrm{CD} 4^{+} \mathrm{T}$ cells is generated, thus inducing rapid spread of HIV-1. This results in a peak of viremia or a viral set point followed by induction of $\mathrm{CD}^{+}$ $\mathrm{T}$ lymphocytes, and dramatic loss of $\mathrm{CD} 4^{+} \mathrm{T}$ cells. Finally the viral load is controlled and maintained at a steady level throughout the chronic phase of HIV-1 infection. Hence, evading the physical barrier posed by the mucosal tissues marks the success of the initial stages of viral infection and spread.

\section{Complement System}

After penetrating the initial mucosal barrier, complement system confers a major host defense mechanism contributing the restriction in viral replication by triggering the recruitment of inflammatory cells and also by rupturing plasma membranes of undesired cells. Complement system functions as inhibitor as well as beneficiary for HIV-1 infection and pathogenesis. Complement pathways lyse HIV-1 particles and the infected cells to neutralize IgG and IgM-bound viruses. However, HIV-1 overcomes the complement mediated inhibition of viral spread or pathogenesis by activating the classical pathway of complement system by binding Clq with envelope protein gp41 [24]. HIV-1 downregulates expression of host complement receptors that impair monocyte chemotactic responses to inflammatory stimuli (exposure of gp120 decreases C5a expression) [25]. Deposition of C3 and C5a facilitates HIV-1 interaction with complement receptor CR3 and CR4 containing cells including monocytes/macrophages and DCs. HIV-1 gp41 interacts with CR3 and this interaction 
enhances both viral entry and viral spread in the cells [26]. HIV-1 also binds to CR1 on erythrocytes and CR2 on B cells and exploits these cells to generate C3d-opsonized infectious HIV-1 reservoirs and to spread infection to the uninfected organs [27-29]. In addition to increasing viral infectivity, HIV-1 gp41 and other viral proteins also stimulate the synthesis of C3 in neurons and astrocytes [30]. This increased production of complements is another means of contributing HIV-1-induced neuropathogenesis. Also, gp41 and gp120 recruit factor $\mathrm{H}$, which is responsible for protecting selfcells from complement mediated lysis to multiple binding sites [31, 32]; thus, in turn, it causes decreased complement dependent lysis of infected cells and virus in vitro [33]. Another complement regulatory factor CD59 present on HIV-1 envelope prevents complement mediated neutralization of antibody bound viruses [34]. Together, these studies indicate that HIV-1 has evolved to override the complement system induced innate antiviral response to increase viral spread.

\section{HIV-1 Induced Changes in Cytokine and Chemokine Profiles}

Cytokines and chemokines have the most influential role in HIV-1 pathogenesis, and the virus exploits the network throughout its life cycle. HIV-1 infection results in the activation of immune cells, altered functions of macrophages, NK cells, and DCs, and the activation of B cells [35-41]. Overactivation of immune cells leads to increased production of proinflammatory anti-inflammatory cytokines and chemokines including IFNs, tumor necrosis factor (TNF)- $\alpha$, interleukin(IL) -1, -2, -4, -8, -6, -10, -15, interferon gamma-induced protein (IP)-10, and monocyte chemotactic protein (MCP)-1 [42, 43]. These cytokines/chemokines either enhance or inhibit HIV-1 replication. With disease progression-a shift from stimulatory Th1 (IL-2, IFN) to inhibitory Th2 (IL-4, IL-10, IL1, IL-6, IL-8, and TNF), cytokine production takes place [44]. Attachment of HIV-1 envelope protein, gp120, triggers production of CC chemokines CCL2, CCL3, CCL4, and CCL5 that are chemoattractants for DCs, macrophages, and lymphocytes. The expression of these $\mathrm{CC}$ chemokines is regulated by cytokines including IL- 6 , TNF- $\alpha$, IL- $1 \beta$, and IL-10, which are also regulated by HIV-1 infection [45]. HIV-1 utilizes different mechanisms to override host cytokine/chemokine networks; for example, HIV-1 transactivator of transcription (Tat) protein mimics $\beta$-chemokines and functions as chemoattractant of monocytes/macrophages that leads to increased activation and infection [46]. A previous study has shown which HIV-1 negative regulatory factor (Nef) increases the production and the stimulatory function of proinflammatory cytokines such as IL-1 $\beta$, IL-12, IL-15, and TNF- $\alpha$ and chemokines such as macrophage inflammatory protein (MIP)- $1 \alpha,-1 \beta$ and IL- 8 when it interacts with immature DCs $[47,48]$. HIV-1 accessory protein viral protein R (Vpr) also alters the level of proinflammatory cytokines [48]. Hence, HIV-1 either by mimicking or by modulating certain cytokines exploits the cytokine network for its replication and survival.

\section{Interferons and Signaling Events}

Among the cytokines, IFNs play the major role in virus infection and confer the first defense. Type I IFNs (include IFN- $\alpha,-\beta,-\varepsilon,-\kappa$, and $-\omega$ ), the innate cytokines produced by innate immune stimuli, have multiple properties including induction of immune activation, enhanced antigen presentation, and antiviral activity [49]. IFNs are mainly produced by IFN regulatory factors (IRFs) especially IRF3 and IRF7 [50]. They bind to IFN receptor IFNAR1/IFNAR2 at the cell surface and form STAT1/STAT2 dimer, which translocates into the nucleus and triggers downstream signaling pathways that result in activation of interferon-stimulated genes (ISGs). Cellular factors, ISGs, generated from type I and type II IFN, induce signaling events that inhibit HIV-1 replication [51, 52]. Recognition of PAMPs stimulates activation of IFN-induced signaling pathways triggering activation of transcriptions factors including NF- $\kappa \mathrm{B}$, activator protein (AP)-1 and IRF3, which in turn leads to production of proinflammatory cytokines and chemokines and antiviral type I IFN. The innate immune response largely depends on virus-induced signaling pathways to induce IFN response. A recent study indicated the role of tripartite motif containing 22 (TRIM22) induced by IFN- $\beta$ as an inhibitor of HIV-1 replication [53].

Since IFN has the most potent role in preventing viral replication and eliminating infection, HIV-1 blocks and/or minimizes the IFN production as well as the downstream signaling pathways to prevent immune responses. However, the mechanism(s) by which HIV-1 can manage to escape IFN induced anti-HIV-1 activity is not fully understood. It was documented that HIV-1 gp120 blocks cytokines including IFN and the cytolytic activity of NK cells by interfering with TLR9 activation in pDCs [54]. In a SIV model of central nervous system, it has been shown that SIV infected astrocytes produce monocyte chemotactic protein (MCP)-1 or CCL2 that binds to the CCR2 receptors on macrophages resulting in suppression of specific ISGs such as TRAIL [55]. In macrophages, HIV-1 exploits another cellular factor, suppressor of cytokine signaling (SOCS) 3 to facilitate its replication. SOCS3 inhibits IFN- $\beta$ signaling in macrophages, and hence it prevents antiviral gene expression that helps HIV-1 replication [56]. Studies also indicated that cellular $3^{\prime}$ repair exonuclease 1 (TREX1) helps the virus to hide from immune activation [57]. The exonuclease activity of TREX1 degrades excess ssDNA of HIV-1 and thus prevents activation of IFN by this excess HIV-1 DNA. Mutation or dysfunction of TREX1 results in accumulation of HIV-1 DNA and triggers DNA sensors. This activates STING, TBK1, and IRF3 transcription factors. Activated IRF3 translocates into nucleus and enhances IFN response by activating the respective promoters in different cell types [57-59].

The release of IFNs leads to the induction of ISG through JAK/STAT pathways. ISGs induce antiviral response. One of the first ISG linked to antiviral response is the IFNinduced protein kinase $\mathrm{R}$ (PKR). It plays an important role, when it binds to dsRNA of viral replication product, forms a dimer by autophosphorylation, and blocks viral replication by inhibiting translation of alpha subunit of elongation 
initiation factor 2 (eIF2 $\alpha$ ) in infected cells. HIV-1 infection does not activate PKR. Virus replicates in cells where a high level of TAR binding protein (TRBP) is present. TRBP functions as a strong inhibitor of PKR and $\operatorname{eIF} 2 \alpha[60,61]$. TRBP also binds to and serves as a cofactor of Dicer, which is required for biosynthesis of microRNA (miRNA) or RNA interference (RNAi) [62], inhibitors of viral replication. HIV-1 overrides the PKR mediated innate response via two different mechanisms. First, HIV-1 Tat RNA binds to TRBP and inhibits PKR activation; also TRBP becomes unavailable for Dicer binding [63]. Thus HIV-1 exploits TRBP as a link between IFN and RNAi mediated antiviral response. Second, HIV-1 Tat also functions as a substrate homologue of eIF2 $\alpha$ and competes for PKR mediated phosphorylation. PKR phosphorylates HIV-1 Tat, and the phosphorylation of Tat is necessary for HIV-1 LTR transactivation [64]. This prevents phosphorylation of eIF $2 \alpha$ and enhances viral replication [65]. Another protein adenosine deaminase acting on RNA (ADAR1) is also reported to inhibit PKR activation [60]. Together, these reports support that HIV-1 proteins play a major role in combating IFN responses, thus blocking innate antiviral activity.

\section{Plasmacytoid Dendritic Cells (pDCs) Function as Major Source of IFN}

After HIV-1 entry into the system, the immediate IFN response is primarily from $\mathrm{pDCs}$. pDCs play the central role in innate immune response against viral pathogens through the secretion of enormous amount of IFN. It expresses TLR7 and TLR9 in addition to CD4, CCR5, and CXCR4 receptors. TLR7 and TLR9 on pDCs bind to ssRNA and unmethylated CpG DNA motif, respectively, and signal through MyD88 that in turn leads to the activation of IRF7 for IFN production. Compared to other blood cells, these cells produce several times more type I IFNs that function as immunostimulatory cytokine to drive mDC maturation [66]. HIV-1 inhibits the innate immune response of $\mathrm{pDCs}$ by reducing $\mathrm{pDC}$ cell counts in peripheral blood. HIV-1 infected individuals are reported to have lower levels of $\mathrm{pDC}$ compared to uninfected individuals [39]. HIV-1 gp120 exposure suppresses pDC activation and production of proinflammatory cytokines mediated through TLR9 [54]. Other studies have demonstrated that HIV-1 blocks pDC function by suppressing TLR7 and TLR8 [67] and also by inhibiting IFN- $\alpha$ [68]. However, before undergoing HIV-1-induced cell death, pDCs upregulate CCR7 production, accumulate in lymph node, and produce high level of IFN [69]. High plasma level of IFN has been observed during acute HIV-1 infection and also during the late stage of the HIV-1 disease. It has also been reported that IFN enhances disease progression to AIDS [70]. HIV-1 induced production of IFN by pDCs leads to the expression of TNF-related apoptosis inducing ligand (TRAIL) [71], which is involved in triggering apoptosis of uninfected $\mathrm{CD}^{+} \mathrm{T}$ cells [72]. Through TLR7, TRAIL turns pDCs into IFNproducing killer pDCs (IKpDCs) that produce high level of TNF- $\alpha$ [73]. It has also been shown that in addition to IFNs activation of $\mathrm{pDCs}, \mathrm{HIV}-1$ also stimulates production of inflammatory cytokines/chemokines including TNF- $\alpha$, IL-6, CXCL10, CCL4, and CCL5 [74, 75].

\section{Functional Dysregulation of Natural Killer (NK) Cells by HIV-1}

The immunomodulatory and cytotoxic activities of NK cells are impaired in HIV-1 infected individuals, diminishing the innate and detrimentally affecting the adaptive immune response [76-79]. Analysis of frequency, phenotypes, and function of peripheral blood CD3-CD56+NK subsets in HIV$1+$ individuals revealed significantly reduced numbers of total NK cells and a striking shift in NK cell subsets. More specifically, CD56 ${ }^{\mathrm{dim}}$ populations of HIV-1 infected subjects were markedly diminished (and exhibited functional abnormalities) when compared to IFN- $\gamma$ producing CD56 ${ }^{\text {bright }}$ NK cell fractions from the same individuals [79]. HIV-1 infection is characterized by a dramatic increase in inhibitory receptors and loss of activating receptors, particularly, NKp30 on NK cells, resulting in loss of NK cell activity and defective crosstalk with DC $[80,81]$. Involvement of HIV-1 in the impairment of $\mathrm{NK}$ cell function in vivo has also been bolstered indicating an association between NK cell ligand HLA-B Bw4-801 and its receptor; the killer immunoglobulinlike receptor (KIR) 3DS1; expression of HLA/KIR subtypes can be linked to efficient NK cell mediated inhibition of HIV1 replication and killing of infected targets $[82,83]$.

The observed NK cell dysregulation is attributed to HIV1 viral proteins $[84,85]$. HIV-1 Nef functions as a potential regulator of $\mathrm{NK}$ cell cytotoxicity due to its involvement in $\mathrm{MHC}$ class I downregulation on $\mathrm{CD}^{+}$cells [86]. The selective downregulation by Nef of HLA A and HLA B but not HLA $\mathrm{C}$ or HLA E molecules in infected target cells inhibits NK cell cytotoxicity which confers an additional evasion strategy targeting the antiviral activities of NK cells [87-89]. HIV-1 Tat inhibits LFA-I-mediated $\mathrm{Ca}^{2+}$ influx through the binding of L-type $\mathrm{Ca}^{2+}$ channel and thereby impairs NK cell cytotoxicity $[90,91]$. A linear motif of HIV-1 gp41 induces expression of NKp44L on $\mathrm{CD}^{+} \mathrm{T}$ cells and results in their depletion by selective lysis by $\mathrm{NK}_{4} 4^{+} \mathrm{NK}$ cells [37]. Azzoni et al. showed that virally suppressed children had normal levels of circulating pDCs, mDC, and total NK cells, yet they had sustained depletion of mature NK cell subsets along with diminished IFN- $\alpha$ production by $\mathrm{pDC}$, which is required to enhance NK cell cytolytic responses [92]. HIV-1 treated target cells exhibit selective upregulation of NKG2D receptor on NK cells, and this upregulation is contact dependent and reversible [93]. Expression of these molecules is directly correlated with efficient killing of infected targets as well as controlling viremia, suggesting that innate immune cells play a critical role in immune control.

\section{Antiviral Host Restriction Factors}

In addition to other innate antiviral factors and innate immune cells, host restriction factors have also been shown to restrict HIV-1 infection in multiple ways. For instance, apolipoprotein B editing catalytic polypeptide (APOBEC3) 
TABLE 1: Role of HIV-1 viral proteins in innate immune evasion.

\begin{tabular}{|c|c|}
\hline HIV-1 accessory proteins & Function in innate immune evasion \\
\hline gp41 & $\begin{array}{l}\text { (i) Activates classical pathway of complement system } \\
\text { (ii) Enhances viral entry and spread through interaction with complement receptor CR3 } \\
\text { (iii) Stimulates synthesis of C3 in neurons and astrocytes } \\
\text { (iv) Recruits factor H responsible for protecting from complement dependent lysis } \\
\text { (v) Induces expression of NKp44L on } \mathrm{CD}^{4+} \mathrm{T} \text { cells and results in their depletion }\end{array}$ \\
\hline gp120 & $\begin{array}{l}\text { (i) Recruits factor } \mathrm{H} \\
\text { (ii) Induces production of inflammatory cytokines and the CC chemokines } \\
\text { (iii) Suppresses pDC activation and produces IFN and other cytokines } \\
\text { (iv) Inhibits cytolytic activity of NK cells by interfering with TLR9 }\end{array}$ \\
\hline Tat & $\begin{array}{l}\text { (i) Substrate homologue for eIF } 2 \alpha \text { and competes for PKR mediated phosphorylation } \\
\text { (ii) Mimics } \beta \text {-chemokines and functions as chemoattractant for monocytes and macrophages } \\
\text { (iii) Inhibits LFA-I mediated } \mathrm{Ca}^{2+} \text { influx and impairs NK cell cytotoxicity } \\
\text { (iv) Interacts with Dicer and inhibits its activity, suppresses miRNA synthesis }\end{array}$ \\
\hline Nef & $\begin{array}{l}\text { (i) Increases expression of proinflammatory cytokines } \\
\text { (ii) Downregulates HLA-A, HLA-B on target cells and inhibits NK cell cytotoxicity } \\
\text { (iii) Helps in budding of HIV-1 } \\
\text { (iv) Produces viral miRNA in HIV-1 persistently infected cells }\end{array}$ \\
\hline Vpr & $\begin{array}{l}\text { (i) Alters the levels of proinflammatory cytokines } \\
\text { (ii) Upregulates NKG2D receptor on NK cells }\end{array}$ \\
\hline Vpu & (i) Causes detachment of viral particle from cell membrane through interaction with tetherin \\
\hline Vif & $\begin{array}{l}\text { (i) Inhibits the packaging of APOBEC } 3 \mathrm{G} \text { in virus producer cells causes proteosomal degradation of } \\
\text { APOBEC } 3 \mathrm{G}\end{array}$ \\
\hline
\end{tabular}

is a member of cytidine deaminase family that has specific anti-HIV-1 property. APOBEC3G incorporates into HIV-1 through interacting with Gag and restricts HIV-1 infection $[94,95]$. It mutates cytidine to uridine in negative sense single stranded DNA by deamination resulting in guanosine to adenosine hypermutation in positive sense viral cDNA and thereby makes it vulnerable to nuclease degradation $[96,97]$. Current studies show that APOBEC3G functions in deaminase independent manner [98]. However, HIV-1 viral infectivity factor (Vif) protein inhibits the packaging of APOBEC3G in virus producer cells by functioning as an adaptor molecule that links a cullin-5-based E3 ubiquitin ligase complex with APOBEC3G leading to polyubiquitination and targeting APOBEC3G and APOBEC3F to proteosomal degradation [99-101].

Another restriction factor implicated in early steps of HIV-1 replication is tripartite motif-containing 5 (TRIM5). TRIM5 functions as E3 ubiquitin ligase and recognizes the motifs in viral capsid in the cytoplasm of an infected cell. TRIM5 prevents reverse transcription and transports viral genome to the nucleus [102]. Although the exact mechanism of TRIM5 inhibition is unknown, it has been suggested that TRIM5 promotes innate immune signaling, which is enhanced by interaction with viral capsid lattice [103]. TRIM5 binds to the viral capsid and activates the protein's ubiquitin ligase activity resulting in the synthesis of ubiquitin chains. These chains stimulate TAK1 (also known as MAP3 K7) phosphorylation followed by expression of NF- $\kappa \mathrm{B}$-dependent genes. In primates, TRIM5 expression regulates expression of genes by AP- 1 and NF- $\kappa$ B activation $[103,104]$.

Tetherin is another intrinsic restriction factor involved in innate antiviral response. Tetherin/BST2, a glycosylated type
II transmembrane protein, prevents budding of newly formed virus particles from plasma membrane of HIV-1 infected cells. Tetherin restricts HIV-1 transmission from one cell to another [105]. Its expression is upregulated by type I IFN. HIV-1 viral protein unique ( $\mathrm{Vpu}$ ) induces detachment of virus particles from cell membrane $[106,107]$. Vpu interacts directly or indirectly with tetherin and reduces its expression at the surface. Viruses that are defective in Vpu expression remain tethered to the cell surface [11]. These tethered virus particles are subjected to endocytosis and degraded in lysosomes [12]. Recent studies indicated that the Vpu-like activity has been reported for other viral proteins including Env and Nef $[13,108,109]$.

\section{Other Regulatory Factors}

Studies have shown that HIV-1 infection as well as innate immune response to viral infection could be controlled by host miRNAs [110]. miRNAs are short $\sim 20-22$ nucleotide long small noncoding RNA molecules expressed in most organisms [111]. These miRNAs control gene expression by binding to the $3^{\prime}$ UTR region of the target mRNAs. They can regulate the expression of both cellular and viral genes. For example, miR-26a, $-34 a, 145$, and let-7b have been reported to regulate IFN- $\beta$ in human and macaque cells [112]. Distinct differences in miRNA profiles have been reported in PBMCs infected with HIV-1 compared to control [113]. HIV-1 replication involves downregulation of specific cellular miRNAs (e.g., miR-29), which plays a significant role in controlling HIV-1 life cycle. Hsa-miR-29 binds to the conserved sequence of Nef $3^{\prime}$-LTR, transports HIV-1 mRNA to P-bodies and inhibits translation of viral mRNA [114]. Other miRNAs 
including miR-150, 223, 198, and 382 have been shown to be downregulated in HIV-1 infected macrophages and are known as anti-HIV-1 miRNAs, as they exert negative effect on the viral replication [115]. Treatment of macrophages with IFN- $\alpha$ and $-\beta$ increases the expression of these miRNAs confirming their role in HIV-1 replication [56]. Alternatively, HIV-1 has also evolved viral miRNA to counterattack the host machineries. HIV-1 Tat interacts with Dicer, inhibits its activity, and suppresses the miRNA synthesis [116]. HIV1 also blocks RNA silencing pathways by TRBP $[62,63]$. Some viral miRNAs such as TAR miRNA, Nef miRNA (miR$\mathrm{N} 367$ ), and $\mathrm{miR}-\mathrm{H} 1$ are expressed, and regulate viral as well as host gene expression to facilitate virus replication and establishment of latency [117].

\section{Conclusion}

The interaction between host and HIV-1 is complex. The host system utilizes its machinery to defend HIV-1 infection, whereas the virus uses the host's tools as means of its own propagation. Starting at the transmission site (mucosal barrier) to immune activation site (lymph node), HIV-1 utilizes very unique strategies such as modification of its PAMPs, rapid mutation in its RNA to escape immune recognition, downregulation of complement receptors, increased secretion of inflammatory factors, and downregulation of NK cell function to overcome the innate immune response. The role of HIV-1 viral proteins to overcome host innate immunity is specified in Table 1. Current research focuses on understanding how HIV-1 overrides the innate immune system at multiple levels. The understanding of how HIV1 prevails the host innate immune defense will increase our knowledge that could improve developing therapeutic approach to resist HIV-1 infection and spreading.

\section{References}

[1] V. Chieux, D. Hober, W. Chehadeh, and P. Wattré, "Anti-viral proteins: from interferon $\alpha$ to its receptor," Annales de Biologie Clinique, vol. 57, no. 3, pp. 283-290, 1999.

[2] G. Hajishengallis, H. Sojar, R. J. Genco, and E. DeNardin, "Intracellular signaling and cytokine induction upon interactions of Porphyromonas gingivalis fimbriae with pattern-recognition receptors," Immunological Investigations, vol. 33, no. 2, pp. 157172, 2004.

[3] V. Racaniello, "Innate immune defenses," 2009, http://www .virology.ws/2009/06/03/innate-immune-defenses/.

[4] A. Takaoka, Z. Wang, M. K. Choi et al., "DAI (DLM-1/ZBP1) is a cytosolic DNA sensor and an activator of innate immune response," Nature, vol. 448, no. 7152, pp. 501-505, 2007.

[5] S. Akira, "TLR signaling," Current Topics in Microbiology and Immunology, vol. 311, pp. 1-16, 2006.

[6] T. Kawai and S. Akira, "Signaling to NF- $\kappa$ B by Toll-like receptors," Trends in Molecular Medicine, vol. 13, no. 11, pp. 460469, 2007.

[7] M. Yoneyama, W. Suhara, Y. Fukuhara, M. Fukuda, E. Nishida, and T. Fujita, "Direct triggering of the type I interferon system by virus infection: activation of a transcription factor complex containing IRF-3 and CBP/p300," EMBO Journal, vol. 17, no. 4, pp. 1087-1095, 1998.

[8] P. M. Pitha, "Innate antiviral response: role in HIV-I infection," Viruses, vol. 3, no. 7, pp. 1179-1203, 2011.

[9] W. de Vries, J. Haasnoot, J. van der Velden et al., "Increased virus replication in mammalian cells by blocking intracellular innate defense responses," Gene Therapy, vol. 15, no. 7, pp. 545552, 2008.

[10] R. A. Lever and A. M. L. Lever, "Intracellular defenses against HIV, viral evasion and novel therapeutic approaches," Journal of the Formosan Medical Association, vol. 110, no. 6, pp. 350-362, 2011.

[11] M. H. Malim and M. Emerman, "HIV-1 accessory proteinsensuring viral survival in a hostile environment," Cell Host and Microbe, vol. 3, no. 6, pp. 388-398, 2008.

[12] T. Goto, S. J. Kennel, M. Abe et al., "A novel membrane antigen selectively expressed on terminally differentiated human B cells," Blood, vol. 84, no. 6, pp. 1922-1930, 1994.

[13] R. Serra-Moreno, B. Jia, M. Breed, X. Alvarez, and D. T. Evans, "Compensatory changes in the cytoplasmic tail of gp41 confer resistance to tetherin/BST-2 in a pathogenic Nef-deleted SIV," Cell Host and Microbe, vol. 9, no. 1, pp. 46-57, 2011.

[14] A. J. McMichael, P. Borrow, G. D. Tomaras, N. Goonetilleke, and B. F. Haynes, "The immune response during acute HIV1 infection: clues for vaccine development," Nature Reviews Immunology, vol. 10, no. 1, pp. 11-23, 2010.

[15] A. Nazli, O. Chan, W. N. Dobson-Belaire et al., "Exposure to HIV-1 directly impairs mucosal epithelial barrier integrity allowing microbial translocation," PLoS Pathogens, vol. 6, no. 4, Article ID e1000852, 2010.

[16] J. M. Brenchley and D. C. Douek, "The mucosal barrier and immune activation in HIV pathogenesis," Current Opinion in HIV and AIDS, vol. 3, no. 3, pp. 356-361, 2008.

[17] B. Kanwar, D. Favre, and J. M. McCune, "Thl7 and regulatory T cells: implications for AIDS pathogenesis," Current Opinion in HIV and AIDS, vol. 5, no. 2, pp. 151-157, 2010.

[18] D. Horejsh, T. J. Ruckwardt, and C. D. Pauza, "CXCR4dependent HIV-1 infection of differentiated epithelial cells," Virus Research, vol. 90, no. 1-2, pp. 275-286, 2002.

[19] C. Agrati, G. D’Offizi, M.-L. Gougeon et al., "Innate gamma/delta T-cells during HIV infection: terra relatively incognita in novel vaccination strategies?" AIDS Reviews, vol. 13, no. 1, pp. 3-12, 2011.

[20] C. A. Derdeyn, J. M. Decker, F. Bibollet-Ruche et al., "Envelopeconstrained neutralization-sensitive HIV-1 after heterosexual transmission," Science, vol. 303, no. 5666, pp. 2019-2022, 2004.

[21] B. F. Keele, E. E. Giorgi, J. F. Salazar-Gonzalez et al., "Identification and characterization of transmitted and early founder virus envelopes in primary HIV-1 infection," Proceedings of the National Academy of Sciences of the United States of America, vol. 105, no. 21, pp. 7552-7557, 2008.

[22] M.-R. Abrahams, J. A. Anderson, E. E. Giorgi et al., "Quantitating the multiplicity of infection with human immunodeficiency virus type 1 subtype $C$ reveals a non-poisson distribution of transmitted variants," Journal of Virology, vol. 83, no. 8, pp. 3556-3567, 2009.

[23] J. F. Salazar-Gonzalez, M. G. Salazar, B. F. Keele et al., "Genetic identity, biological phenotype, and evolutionary pathways of transmitted/founder viruses in acute and early HIV-1 infection," 
Journal of Experimental Medicine, vol. 206, no. 6, pp. 1273-1289, 2009.

[24] P. Marschang, C. F. Ebenbichler, and M. P. Dierich, "HIV and complement: role of the complement system in HIV infection," International Archives of Allergy and Immunology, vol. 103, no. 2, pp. 113-117, 1994.

[25] C. Speth and M. P. Dierich, "Modulation of cell surface protein expression by infection with HIV-1," Leukemia, vol. 13, supplement 1, pp. S99-S105, 1999.

[26] H. Stoiber, I. Frank, M. Spruth et al., "Inhibition of HIV-1 infection in vitro by monoclonal antibodies to the complement receptor type 3 (CR3): an accessory role for CR3 during virus entry?" Molecular Immunology, vol. 34, no. 12-13, pp. 855-863, 1997.

[27] E. Horakova, O. Gasser, S. Sadallah et al., "Complement mediates the binding of HIV to erythrocytes," Journal of Immunology, vol. 173, no. 6, pp. 4236-4241, 2004.

[28] Z. Bánki, D. Wilflingseder, C. G. Ammann et al., "Factor I-mediated processing of complement fragments on HIV immune complexes targets HIV to CR2-expressing B cells and facilitates B cell-mediated transmission of opsonized HIV to T cells," Journal of Immunology, vol. 177, no. 5, pp. 3469-3476, 2006.

[29] S. Döpper, D. Wilflingseder, W. M. Prodinger et al., "Mechanism(s) promoting HIV-1 infection of primary unstimulated T lymphocytes in autologous B cell/T cell co-cultures," European Journal of Immunology, vol. 33, no. 8, pp. 2098-2107, 2003.

[30] C. Speth, T. Schabetsberger, I. Mohsenipour et al., "Mechanism of human immunodeficiency virus-induced complement expression in astrocytes and neurons," Journal of Virology, vol. 76, no. 7, pp. 3179-3188, 2002.

[31] C. Pinter, A. G. Siccardi, L. Lopalco, R. Longhi, and A. Clivio, "HIV glycoprotein 41 and complement factor $\mathrm{H}$ interact with each other and share functional as well as antigenic homology," AIDS Research and Human Retroviruses, vol. 11, no. 8, pp. 971980, 1995.

[32] H. Stoiber, C. Ebenbichler, R. Schneider, J. Janatova, and M. P. Dierich, "Interaction of several complement proteins with gp120 and gp41, the two envelope glycoproteins of HIV-1," AIDS, vol. 9, no. 1, pp. 19-26, 1995.

[33] H. Stoiber, C. Pintér, A. G. Siccardi, A. Clivio, and M. P. Dierich, "Efficient destruction of human immunodeficiency virus in human serum by inhibiting the protective action of complement factor $\mathrm{H}$ and decay accelerating factor (DAF, CD55)," Journal of Experimental Medicine, vol. 183, no. 1, pp. 307-310, 1996.

[34] J. Schmitz, J. P. Zimmer, B. Kluxen et al., "Antibody-dependent complement-mediated cytotoxicity in sera from patients with HIV-1 infection is controlled by CD55 and CD59," Journal of Clinical Investigation, vol. 96, no. 3, pp. 1520-1526, 1995.

[35] D. C. Douek, L. J. Picker, and R. A. Koup, “T cell dynamics in HIV-1 infection," Annual Review of Immunology, vol. 21, pp. 265-304, 2003.

[36] S. M. Wahl, T. Greenwell-Wild, and N. Vázquez, "HIV accomplices and adversaries in macrophage infection," Journal of Leukocyte Biology, vol. 80, no. 5, pp. 973-983, 2006.

[37] V. Vieillard, J. L. Strominger, and P. Debré, "NK cytotoxicity against $\mathrm{CD}^{+} \mathrm{T}$ cells during HIV-1 infection: a gp41 peptide induces the expression of an NKp44 ligand," Proceedings of the
National Academy of Sciences of the United States of America, vol. 102, no. 31, pp. 10981-10986, 2005.

[38] B. He, X. Qiao, P. J. Klasse et al., "HIV-1 envelope triggers polyclonal Ig class switch recombination through a CD40independent mechanism involving BAFF and C-type lectin receptors," Journal of Immunology, vol. 176, no. 7, pp. 3931-3941, 2006.

[39] M. Müller-Trutwin and A. Hosmalin, "Role for plasmacytoid dendritic cells in anti-HIV innate immunity," Immunology and Cell Biology, vol. 83, no. 5, pp. 578-585, 2005.

[40] M. Van Der Vlist, A. M. G. Van Der Aar, S. I. Gringhuis, and T. B. H. Geijtenbeek, "Innate signaling in HIV-1 infection of dendritic cells," Current Opinion in HIV and AIDS, vol. 6, no. 5, pp. 348-352, 2011.

[41] S. R. Walsh, N. Bhardwaj, and R. T. Gandhil, "Dendritic cells and the promise of therapeutic vaccines for human immunodeficiency virus (HIV)-1," Current HIV Research, vol. 1, no. 2, pp. 205-216, 2003.

[42] A. R. Stacey, P. J. Norris, L. Qin et al., "Induction of a striking systemic cytokine cascade prior to peak viremia in acute human immunodeficiency virus type 1 infection, in contrast to more modest and delayed responses in acute hepatitis B and $\mathrm{C}$ virus infections," Journal of Virology, vol. 83, no. 8, pp. 3719-3733, 2009.

[43] J. Rychert, D. Strick, S. Bazner, J. Robinson, and E. Rosenberg, "Detection of HIV gp120 in plasma during early HIV infection is associated with increased proinflammatory and immunoregulatory cytokines," AIDS Research and Human Retroviruses, vol. 26, no. 10, pp. 1139-1145, 2010.

[44] K. Kedzierska and S. M. Crowe, "Cytokines and HIV-1: interactions and clinical implications," Antiviral Chemistry and Chemotherapy, vol. 12, no. 3, pp. 133-150, 2001.

[45] L. Fantuzzi, F. Belardelli, and S. Gessani, "Monocyte/macrophage-derived CC chemokines and their modulation by HIV1 and cytokines: a complex network of interactions influencing viral replication and AIDS pathogenesis," Journal of Leukocyte Biology, vol. 74, no. 5, pp. 719-725, 2003.

[46] A. Albini, S. Ferrini, R. Benelli et al., "HIV-1 Tat protein mimicry of chemokines," Proceedings of the National Academy of Sciences of the United States of America, vol. 95, no. 22, pp. 13153-13158, 1998.

[47] M. Giovanna Quaranta, E. Tritarelli, L. Giordani, and M. Viora, "HIV-1 Nef induces dendritic cell differentiation: a possible mechanism of uninfected CD4(+) T cell activation," Experimental Cell Research, vol. 275, no. 2, pp. 243-254, 2002.

[48] D. Guha, P. Nagilla, C. Redinger, A. Srinivasan et al., "Neuronal apoptosis by HIV-1 Vpr: contribution of proinflammatory molecular networks from infected target cells," Journal of Neuroinflammation, vol. 9, article 138, 2012.

[49] J. K. Yamamoto, F. Barre-Sinoussi, and V. Bolton, "Human alpha- and beta-interferon but not gamma-suppress the in vitro replication of LAV, HTLV-III, and ARV-2," Journal of Interferon Research, vol. 6, no. 2, pp. 143-152, 1986.

[50] W. Chen and J. Royer William E., "Structural insights into interferon regulatory factor activation," Cellular Signalling, vol. 22, no. 6, pp. 883-887, 2010.

[51] P. M. Pitha, "Multiple effects of interferon on the replication of human immunodeficiency virus type 1," Antiviral Research, vol. 24, no. 2-3, pp. 205-219, 1994. 
[52] W. Hou, X. Wang, L. Ye et al., "Lambda interferon inhibits human immunodeficiency virus type 1 infection of macrophages," Journal of Virology, vol. 83, no. 8, pp. 3834-3842, 2009.

[53] S. D. Barr, J. R. Smiley, and F. D. Bushman, "The interferon response inhibits HIV particle production by induction of TRIM22," PLoS Pathogens, vol. 4, no. 2, Article ID e1000007, 2008.

[54] E. Martinelli, C. Cicala, D. Van Ryk et al., "HIV-1 gp120 inhibits TLR9-mediated activation and IFN- $\alpha$ secretion in plasmacytoid dendritic cells," Proceedings of the National Academy of Sciences of the United States of America, vol. 104, no. 9, pp. 33963401, 2007.

[55] L. A. Zaritsky, L. Gama, and J. E. Clements, "Canonical type I IFN signaling in simian immunodeficiency virus-infected macrophages is disrupted by astrocyte-secreted CCL2," Journal of Immunology, vol. 188, no. 8, pp. 3876-3885, 2012.

[56] V. Cobos Jimenez, T. Booiman, S. W. de Taeye et al., "Differential expression of HIV-1 interfering factors in monocyte-derived macrophages stimulated with polarizing cytokines or interferons," Scientific Reports, vol. 2, article 763, 2012.

[57] N. Yan, A. D. Regalado-Magdos, B. Stiggelbout, M. A. LeeKirsch, and J. Lieberman, "The cytosolic exonuclease TREX1 inhibits the innate immune response to human immunodeficiency virus type 1," Nature Immunology, vol. 11, no. 11, pp. 10051013, 2010.

[58] A. Lepelley, S. Louis, M. Sourisseau et al., "Innate sensing of HIV-infected cells," PLoS Pathogens, vol. 7, no. 2, Article ID e1001284, 2011.

[59] T. B. H. Geijtenbeek, "Host DNase TREX1 hides HIV from DNA sensors," Nature Immunology, vol. 11, no. 11, pp. 979-980, 2010.

[60] G. Clerzius, J.-F. Gélinas, and A. Gatignol, "Multiple levels of PKR inhibition during HIV-1 replication," Reviews in Medical Virology, vol. 21, no. 1, pp. 42-53, 2011.

[61] H. Park, M. V. Davies, J. O. Langland et al., “TAR RNA-binding protein is an inhibitor of the interferon-induced protein kinase PKR," Proceedings of the National Academy of Sciences of the United States of America, vol. 91, no. 11, pp. 4713-4717, 1994.

[62] T. P. Chendrimada, R. I. Gregory, E. Kumaraswamy et al., "TRBP recruits the Dicer complex to Ago2 for microRNA processing and gene silencing," Nature, vol. 436, no. 7051, pp. 740-744, 2005.

[63] A. Gatignol, S. Laine, and G. Clerzius, "Dual role of TRBP in HIV replication and RNA interference: viral diversion of a cellular pathway or evasion from antiviral immunity?" Retrovirology, vol. 2, article 65, 2005.

[64] L. Endo-Munoz, T. Warby, D. Harrich, and N. A. J. McMillan, "Phosphorylation of HIV Tat by PKR increases interaction with TAR RNA and enhances transcription," Virology Journal, vol. 2, article 17, 2005.

[65] S. R. Brand, R. Kobayashi, and M. B. Mathews, "The Tat protein of human immunodeficiency virus type 1 is a substrate and inhibitor of the interferon-induced, virally activated protein kinase, PKR," Journal of Biological Chemistry, vol. 272, no. 13, pp. 8388-8395, 1997.

[66] J.-F. Fonteneau, M. Larsson, A.-S. Beignon et al., "Human immunodeficiency virus type 1 activates plasmacytoid dendritic cells and concomitantly induces the bystander maturation of myeloid dendritic cells," Journal of Virology, vol. 78, no. 10, pp. 5223-5232, 2004.
[67] J. A. Martinson, A. Roman-Gonzalez, A. R. Tenorio et al., "Dendritic cells from HIV-1 infected individuals are less responsive to toll-like receptor (TLR) ligands," Cellular Immunology, vol. 250, no. 1-2, pp. 75-84, 2007.

[68] J. C. Tilton, M. M. Manion, M. R. Luskin et al., "Human immunodeficiency virus viremia induces plasmacytoid dendritic cell activation in vivo and diminished alpha interferon production in vitro," Journal of Virology, vol. 82, no. 8, pp. 3997-4006, 2008.

[69] C. Lehmann, M. Lafferty, A. Garzino-Demo et al., "Plasmacytoid dendritic cells accumulate and secrete interferon alpha in lymph nodes of HIV-1 patients," PloS ONE, vol. 5, no. 6, Article ID el1110, 2010.

[70] J. E. Ho, S. G. Deeks, F. M. Hecht et al., "Initiation of antiretroviral therapy at higher nadir $\mathrm{CD} 4^{+} \mathrm{T}$-cell counts is associated with reduced arterial stiffness in HIV-infected individuals," AIDS, vol. 24, no. 12, pp. 1897-1905, 2010.

[71] J.-P. Herbeuval, A. Boasso, J.-C. Grivel et al., "TNF-related apoptosis-inducing ligand (TRAIL) in HIV-1-infected patients and its in vitro production by antigen-presenting cells," Blood, vol. 105, no. 6, pp. 2458-2464, 2005.

[72] M. Lichtner, C. Marañón, P.-O. Vidalain et al., "HIV type 1infected dendritic cells induce apoptotic death in infected and uninfected primary CD $4^{+}$T lymphocytes," AIDS Research and Human Retroviruses, vol. 20, no. 2, pp. 175-182, 2004.

[73] A. W. Hardy, D. R. Graham, G. M. Shearer, and J.-P. Herbeuval, "HIV turns plasmacytoid dendritic cells (pDC) into TRAILexpressing killer pDC and down-regulates HIV coreceptors by Toll-like receptor 7-induced IFN- $\alpha$," Proceedings of the National Academy of Sciences of the United States of America, vol. 104, no. 44, pp. 17453-17458, 2007.

[74] N. J. Megjugorac, H. A. Young, S. B. Amrute, S. L. Olshalsky, and P. Fitzgerald-Bocarsly, "Virally stimulated plasmacytoid dendritic cells produce chemokines and induce migration of $\mathrm{T}$ and NK cells," Journal of Leukocyte Biology, vol. 75, no. 3, pp. 504-514, 2004.

[75] G. Penna, M. Vulcano, S. Sozzani, and L. Adorini, "Differential migration behavior and chemokine production by myeloid and plasmacytoid dendritic cells," Human Immunology, vol. 63, no. 12, pp. 1164-1171, 2002.

[76] G. Alter, N. Teigen, B. T. Davis et al., "Sequential deregulation of NK cell subset distribution and function starting in acute HIV-1 infection," Blood, vol. 106, no. 10, pp. 3366-3369, 2005.

[77] G. Alter, T. J. Suscovich, M. Kleyman et al., "Low perforin and elevated SHIP-1 expression is associated with functional anergy of natural killer cells in chronic HIV-1 infection," AIDS, vol. 20, no. 11, pp. 1549-1551, 2006.

[78] M. L. LaBonte, P. F. McKay, and N. L. Letvin, "Evidence of NK cell dysfunction in SIV-infected rhesus monkeys: impairment of cytokine secretion and NKG2C/C2 expression," European Journal of Immunology, vol. 36, no. 9, pp. 2424-2433, 2006.

[79] U.-C. Meier, R. E. Owen, E. Taylor et al., "Shared alterations in NK cell frequency, phenotype, and function in chronic human immunodeficiency virus and hepatitis C virus infections," Journal of Virology, vol. 79, no. 19, pp. 12365-12374, 2005.

[80] D. Mavilio, G. Lombardo, A. Kinter et al., "Characterization of the defective interaction between a subset of natural killer cells and dendritic cells in HIV-1 infection," Journal of Experimental Medicine, vol. 203, no. 10, pp. 2339-2350, 2006.

[81] A. De Maria, M. Fogli, P. Costa et al., "The impaired NK cell cytolytic function in viremic HIV-1 infection is associated with 
a reduced surface expression of natural cytotoxicity receptors (NKp46, NKp30 and NKp44)," European Journal of Immunology, vol. 33, no. 9, pp. 2410-2418, 2003.

[82] M. P. Martin, Y. Qi, X. Gao et al., "Innate partnership of HLA-B and KIR3DL1 subtypes against HIV-1," Nature Genetics, vol. 39, no. 6, pp. 733-740, 2007.

[83] G. Alter, M. P. Martin, N. Teigen et al., "Differential natural killer cell-mediated inhibition of HIV-1 replication based on distinct KIR/HLA subtypes," Journal of Experimental Medicine, vol. 204, no. 12, pp. 3027-3036, 2007.

[84] M. T. M. Vossen, E. M. Westerhout, C. Söderberg-Nauclér, and E. J. H. J. Wiertz, "Viral immune evasion: a masterpiece of evolution," Immunogenetics, vol. 54, no. 8, pp. 527-542, 2002.

[85] A. Ahmad and R. Ahmad, "HIV's evasion of host's NK cell response and novel ways of its countering and boosting antiHIV immunity," Current HIV Research, vol. 1, no. 3, pp. 295-307, 2003.

[86] K. L. Collins and D. Baltimore, "HIV's evasion of the cellular immune response," Immunological Reviews, vol. 168, pp. 65-74, 1999.

[87] G. B. Cohen, R. T. Gandhi, D. M. Davis et al., "The selective downregulation of class I major histocompatibility complex proteins by HIV-1 protects HIV-infected cells from NK cells," Immunity, vol. 10, no. 6, pp. 661-671, 1999.

[88] T. Swigut, A. J. Iafrate, J. Muench, F. Kirchhoff, and J. Skowronski, "Simian and human immunodeficiency virus Nef proteins use different surfaces to downregulate class I major histocompatibility complex antigen expression," Journal of Virology, vol. 74, no. 12, pp. 5691-5701, 2000.

[89] J. Nattermann, H. D. Nischalke, V. Hofmeister et al., "HIV1 infection leads to increased HLA-E expression resulting in impaired function of natural killer cells," Antiviral Therapy, vol. 10, no. 1, pp. 95-107, 2005.

[90] M. R. Zocchi, A. Rubartelli, P. Morgavi, and A. Poggi, "HIV-1 tat inhibits human natural killer cell function by blocking Ltype calcium channels," Journal of Immunology, vol. 161, no. 6, pp. 2938-2943, 1998.

[91] A. Poggi, R. Carosio, G. M. Spaggiari et al., "NK cell activation by dendritic cells is dependent on LFA-1-mediated induction of calcium-calmodulin kinase II: inhibition by HIV-1 Tat Cterminal domain," Journal of Immunology, vol. 168, no. 1, pp. 95-101, 2002.

[92] L. Azzoni, R. M. Rutstein, J. Chehimi, M. A. Farabaugh, A. Nowmos, and L. J. Montaner, "Dendritic and natural killer cell subsets associated with stable or declining $\mathrm{CD} 4^{+}$cell counts in treated HIV-1-infected children," Journal of Infectious Diseases, vol. 191, no. 9, pp. 1451-1459, 2005.

[93] J. Richard, S. Sindhu, T. N. Q. Pham, J.-P. Belzile, and É. A. Cohen, "HIV-1 Vpr up-regulates expression of ligands for the activating NKG2D receptor and promotes NK cell-mediated killing," Blood, vol. 115, no. 7, pp. 1354-1363, 2010.

[94] M. Douaisi, S. Dussart, M. Courcoul, G. Bessou, R. Vigne, and E. Decroly, "HIV-1 and MLV Gag proteins are sufficient to recruit APOBEC3G into virus-like particles," Biochemical and Biophysical Research Communications, vol. 321, no. 3, pp. 566573, 2004.

[95] V.Zennou, D. Perez-Caballero, H. Göttlinger, and P. D. Bieniasz, "APOBEC3G incorporation into human immunodeficiency virus type 1 particles," Journal of Virology, vol. 78, no. 21, pp. 12058-12061, 2004.
[96] R. S. Harris, K. N. Bishop, A. M. Sheehy et al., "DNA deamination mediates innate immunity to retroviral infection," Cell, vol. 113, no. 6, pp. 803-809, 2003.

[97] B. Mangeat, P. Turelli, G. Caron, M. Friedli, L. Perrin, and D. Trono, "Broad antiretroviral defence by human APOBEC3G through lethal editing of nascent reverse transcripts," Nature, vol. 424, no. 6944, pp. 99-103, 2003.

[98] E. N. C. Newman, R. K. Holmes, H. M. Craig et al., "Antiviral function of APOBEC $3 \mathrm{G}$ can be dissociated from cytidine deaminase activity," Current Biology, vol. 15, no. 2, pp. 166-170, 2005.

[99] M. Marin, K. M. Rose, S. L. Kozak, and D. Kabat, "HIV-1 Vif protein binds the editing enzyme APOBEC3G and induces its degradation," Nature Medicine, vol. 9, no. 11, pp. 1398-1403, 2003.

[100] X. Yu, Y. Yu, B. Liu et al., "Induction of APOBEC3G ubiquitination and degradation by an HIV-1 Vif-Cul5-SCF complex," Science, vol. 302, no. 5647, pp. 1056-1060, 2003.

[101] A. Mehle, B. Strack, P. Ancuta, C. Zhang, M. McPike, and D. Gabuzda, "Vif overcomes the innate antiviral activity of APOBEC3G by promoting its degradation in the ubiquitinproteasome pathway," Journal of Biological Chemistry, vol. 279, no. 9, pp. 7792-7798, 2004.

[102] S. de Silva and L. Wu, "TRIM5 acts as more than a retroviral restriction factor," Viruses, vol. 3, no. 7, pp. 1204-1209, 2011.

[103] T. Pertel, S. Hausmann, D. Morger et al., "TRIM5 is an innate immune sensor for the retrovirus capsid lattice," Nature, vol. 472, no. 7343, pp. 361-365, 2011.

[104] C. Aiken and S. Joyce, "Immunology: TRIM5 does double duty," Nature, vol. 472, no. 7343, pp. 305-306, 2011.

[105] B. D. Kuhl, R. D. Sloan, D. A. Donahue, T. Bar-Magen, C. Liang, and M. A. Wainberg, "Tetherin restricts direct cell-tocell infection of HIV-1," Retrovirology, vol. 7, article 115, 2010.

[106] S. J. D. Neil, S. W. Eastman, N. Jouvenet, and P. D. Bieniasz, "HIV-1 Vpu promotes release and prevents endocytosis of nascent retrovirus particles from the plasma membrane," PLoS Pathogens, vol. 2, no. 5, article e39, 2006.

[107] L. Rong, J. Zhang, J. Lu et al., "The transmembrane domain of BST-2 determines its sensitivity to down-modulation by human immunodeficiency virus type $1 \mathrm{Vpu}$, Journal of Virology, vol. 83, no. 15, pp. 7536-7546, 2009.

[108] B. Jia, R. Serra-Moreno, W. Neidermyer Jr. et al., "Speciesspecific activity of SIV Nef and HIV-1 Vpu in overcoming restriction by tetherin/BST2," PLoS Pathogens, vol. 5, no. 5, Article ID e1000429, 2009.

[109] F. Zhang, S. J. Wilson, W. C. Landford et al., "Nef proteins from simian immunodeficiency viruses are tetherin antagonists," Cell Host and Microbe, vol. 6, no. 1, pp. 54-67, 2009.

[110] R. Triboulet, B. Mari, Y.-L. Lin et al., "Suppression of MicroRNA-silencing pathway by HIV-1 during virus replication," Science, vol. 315, no. 5818, pp. 1579-1582, 2007.

[111] D. P. Bartel, "MicroRNAs: genomics, biogenesis, mechanism, and function," Cell, vol. 116, no. 2, pp. 281-297, 2004.

[112] K. W. Witwer, J. M. Sisk, L. Gama, and J. E. Clements, "MicroRNA regulation of IFN- $\beta$ protein expression: rapid and sensitive modulation of the innate immune response," Journal of Immunology, vol. 184, no. 5, pp. 2369-2376, 2010.

[113] A. Gupta, P. Nagilla, H.-S. Le et al., "Comparative expression profile of miRNA and mRNA In primary peripheral blood 
mononuclear cells infected with human immunodeficiency virus (HIV-1)," PloS ONE, vol. 6, no. 7, Article ID e22730, 2011.

[114] G. Sun, H. Li, X. Wu et al., "Interplay between HIV-1 infection and host microRNAs," Nucleic Acids Research, vol. 40, no. 5, pp. 2181-2196, 2012.

[115] J. M. Sisk, J. E. Clements, and K. W. Witwer, "miRNA profiles of monocyte-lineage cells are consistent with complicated roles in HIV-1 restriction," Viruses, vol. 4, no. 10, pp. 1844-1864, 2012.

[116] Y. Bennasser, S.-Y. Le, M. Benkirane, and K.-T. Jeang, "Evidence that HIV-1 encodes an siRNA and a suppressor of RNA silencing," Immunity, vol. 22, no. 5, pp. 607-619, 2005.

[117] A. Narayanan, K. Kehn-Hall, C. Bailey, and F. Kashanchi, "Analysis of the roles of HIV-derived microRNAs," Expert Opinion on Biological Therapy, vol. 11, no. 1, pp. 17-29, 2011. 


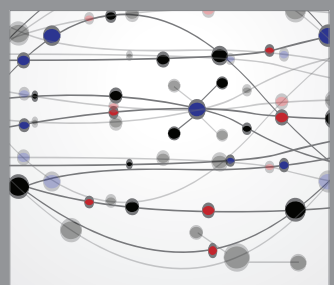

The Scientific World Journal
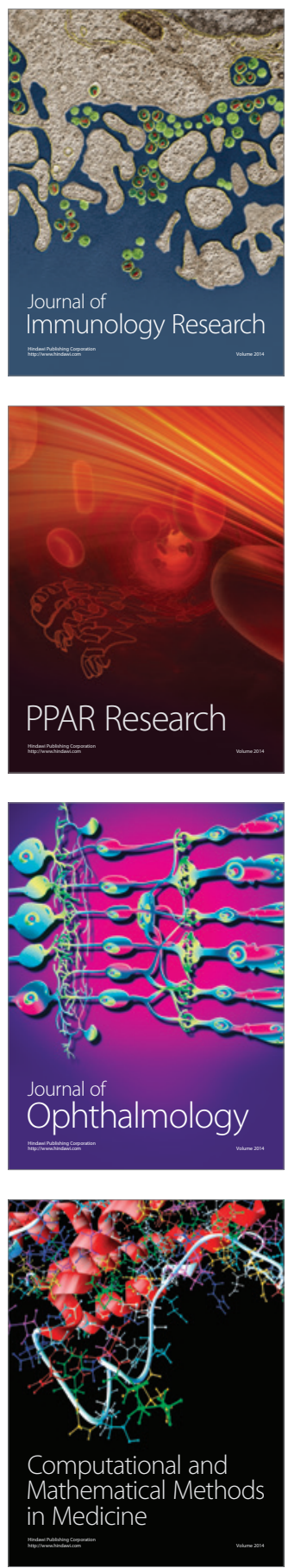

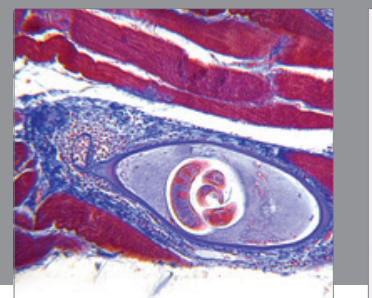

Gastroenterology

Research and Practice
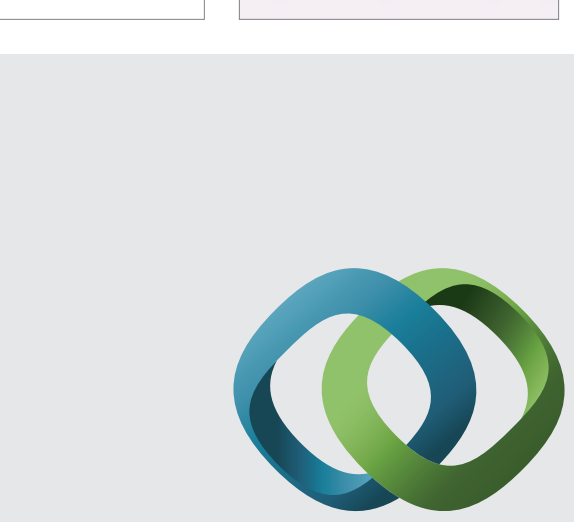

\section{Hindawi}

Submit your manuscripts at

http://www.hindawi.com
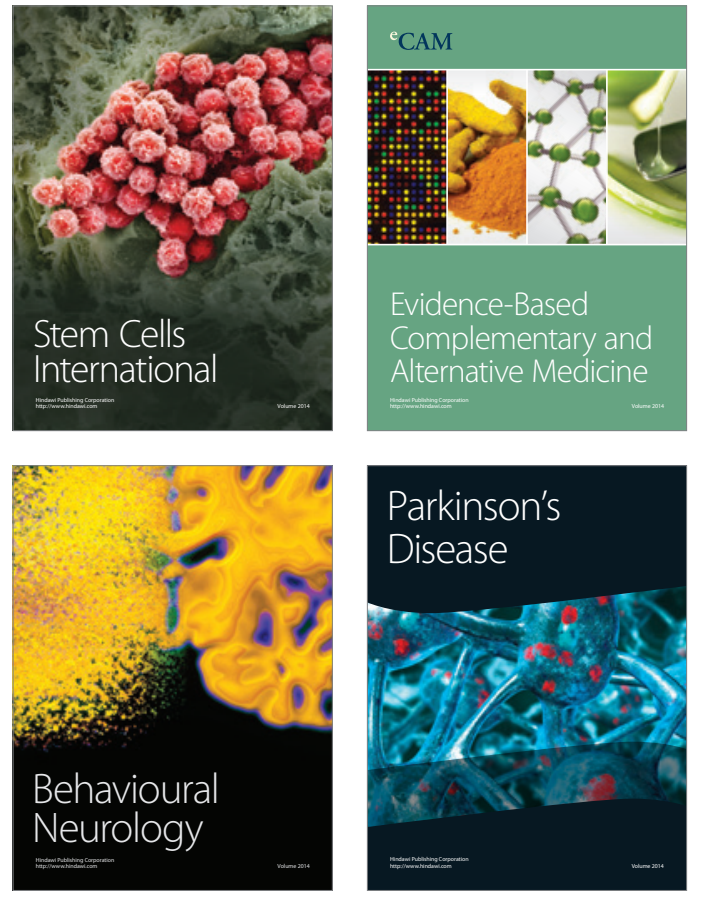
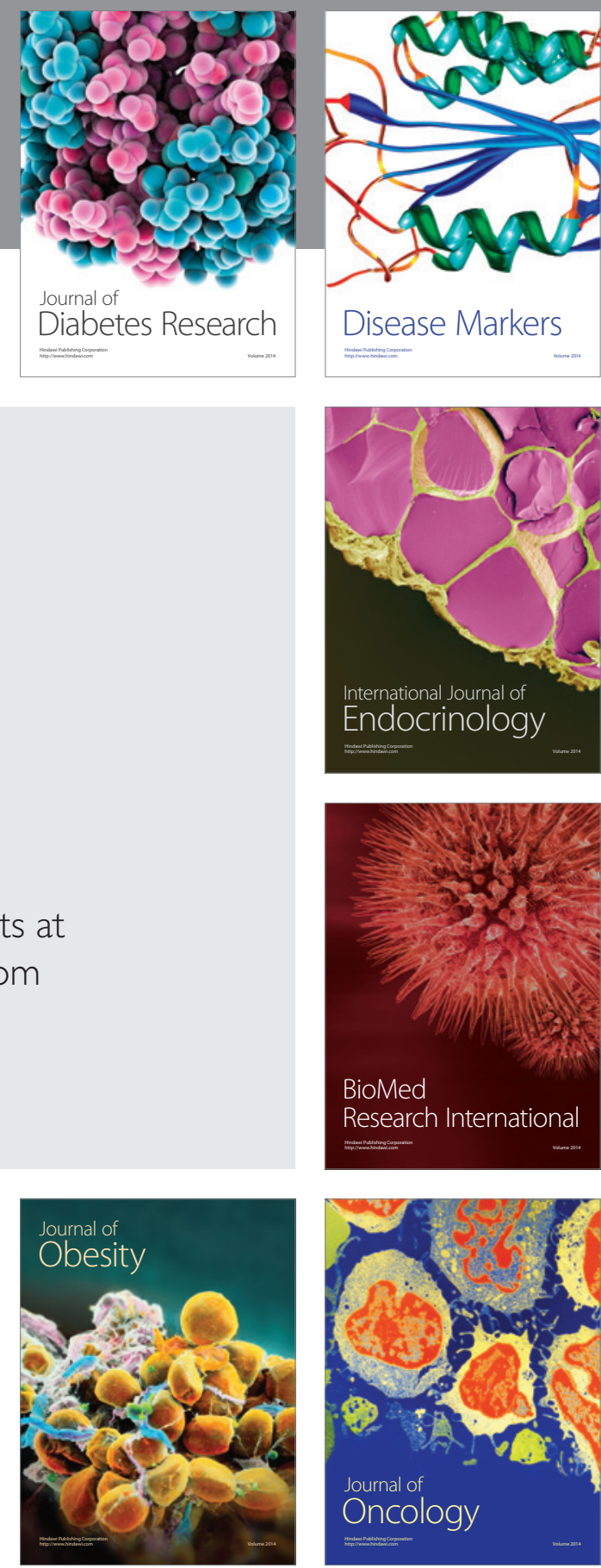

Disease Markers
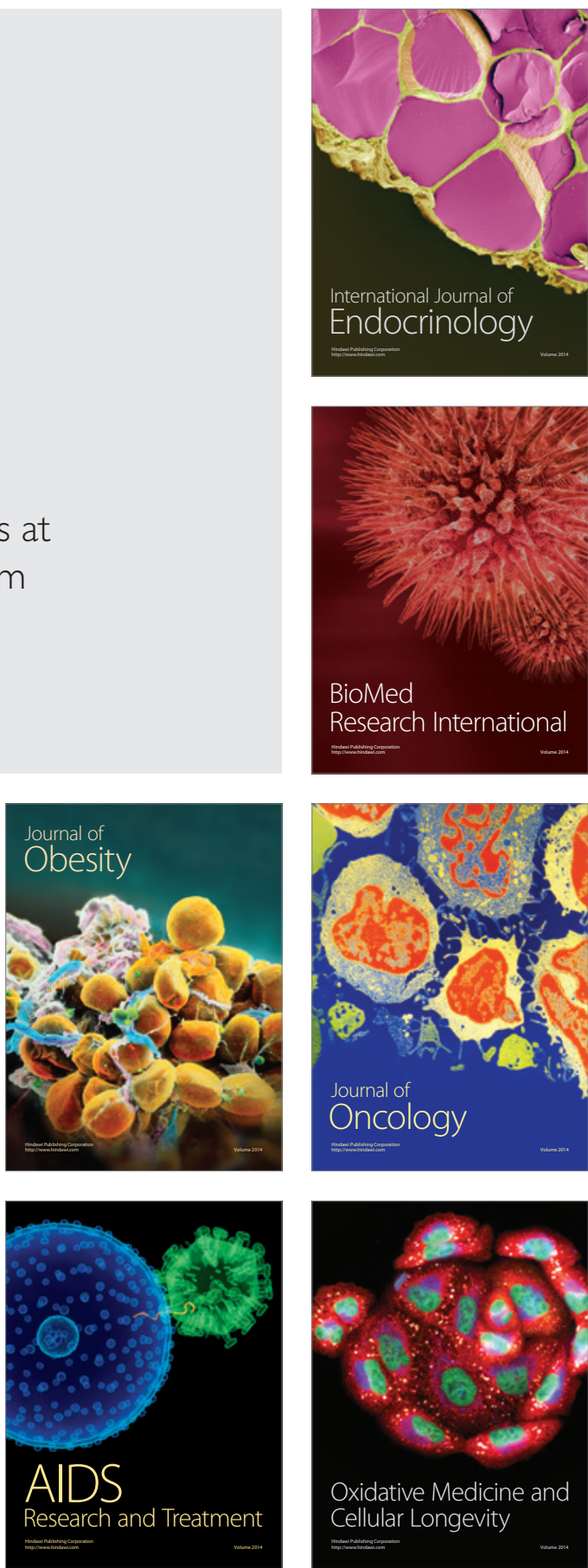